\title{
Multi-Omic Prediction of Overall Survival in Patients With Glioblastoma: Additive and Synergistic Value of Clinical Measures, Radiomics, and Genomics
}

\section{Anahita Fathi Kazerooni}

University of Pennsylvania

\section{Sanjay Saxena}

University of Pennsylvania

\section{Erik Toorens}

University of Pennsylvania

Danni Tu

University of Pennsylvania

\section{Vishnu Bashyam}

University of Pennsylvania

Hamed Akbari

University of Pennsylvania

\section{Elizabeth Mamourian}

University of Pennsylvania

\section{Chiharu Sako}

University of Pennsylvania

Costas Koumenis

University of Pennsylvania loannis Verginadis

University of Pennsylvania

\section{Ragini Verma}

University of Pennsylvania

\section{Russell T. Shinohara}

University of Pennsylvania

Arati S. Desai

University of Pennsylvania

Robert A. Lustig

University of Pennsylvania

\section{Steven Brem}

University of Pennsylvania

\section{Suyash Mohan}


University of Pennsylvania

\section{Stephen J. Bagley}

University of Pennsylvania

\section{Tapan Ganguly}

University of Pennsylvania

Donald M. O'Rourke

University of Pennsylvania

\section{Spyridon Bakas}

University of Pennsylvania

MacLean P. Nasrallah

University of Pennsylvania

Christos Davatzikos ( $\sim$ Christos.Davatzikos@pennmedicine.upenn.edu )

University of Pennsylvania

\section{Research Article}

Keywords: Glioblastoma, survival, multi-omics, radiomics, genomics

Posted Date: September 22nd, 2021

DOl: https://doi.org/10.21203/rs.3.rs-908405/v1

License: (c) (i) This work is licensed under a Creative Commons Attribution 4.0 International License. Read Full License 


\section{Abstract}

Background. Multi-omic data, i.e., clinical measures, radiomic, and genetic data, capture multi-faceted tumor characteristics, contributing to a comprehensive patient risk assessment. Here, we investigate the additive value and independent reproducibility of integrated diagnostics in prediction of overall survival (OS) in newly diagnosed, treatment-naïve, IDH-wildtype GBM patients, by combining conventional and deep learning methods.

Methods. Conventional radiomics and deep learning features were extracted from pre-operative multiparametric MRI of 516 GBM patients. SVM classifiers were trained on the discovery cohort $(n=404)$ to categorize patient groups of high-risk (OS<6 months) vs all, and low-risk (OS $\geq 18$ months) vs all. The trained patient stratification model was independently tested in the replication cohort $(n=112)$ and a patient-wise survival prediction index ( $\mathrm{SPI}_{\text {radiomics }}$ ) was produced. Multivariate Cox-PH models were generated for the replication cohort, first based on clinical measures solely, and then by layering on radiomics and molecular information.

Results. Evaluation of the high-risk and low-risk classifiers in the discovery/replication cohorts revealed AUCs of 0.78 (95\%Cl:0.70-0.85)/ 0.75 (95\%Cl:0.64-0.79) and 0.75 (95\% Cl: $0.65-0.84) / 0.63(95 \% \mathrm{Cl}$ : 0.52-0.71), respectively. Cox-PH modeling showed a concordance index of 0.65 (95\% Cl:0.6-0.7) for clinical data, 0.70 (95\% Cl:0.65-0.75) for clinical and radiomics, $0.72(95 \% \mathrm{Cl}: 0.68-0.77)$ for clinical, MGMT methylation, and radiomics, and 0.75 (95\% Cl:0.72-0.79) for the combination of all omics, i.e., clinical, MGMT methylation, radiomics, and genomics.

Conclusions. This study signifies the value of integrated diagnostics for improved prediction of OS in GBM. Our multi-omic survival prediction tool is easily scalable and can be used for more effective clinical trial stratification.

\section{Introduction}

Glioblastoma (GBM) is the most common and aggressive primary brain neoplasm in adults with a dismal prognosis. Standard treatment consists of maximal safe surgical resection followed by radiation therapy concomitant with temozolomide (TMZ) chemotherapy, which yields a median overall survival (OS) of 14.6 to 16.7 months ${ }^{1}$. While GBM is nearly always fatal, there is overwhelming evidence that the prognosis of patients with GBM varies with patient age 2; clinical features [ECOG score, addition of temozolomide, bilateral spread], performance score ${ }^{2}$, extent of surgical resection ${ }^{2,3}$, and molecular characteristics ${ }^{4}$, e.g., the mutational status of the isocitrate dehydrogenase (IDH) genes, and the methylation status of the $O^{6}$-methylguanine-DNA methyltransferase (MGMT) promoter ${ }^{5}$. However, accurate determination of $M G M T$ methylation status is limited by several factors, such as inter-observer variability, assessment technique, and cutoff levels, imposing a challenge on multi-institutional clinical trials ${ }^{6-8}$. Furthermore, longer OS has been reported in a subset of patients with unmethylated MGMT, adding complexity to prediction of survival, and a personalized, precision approach to the patient 5 . 
The advent of next generation sequencing (NGS) over a decade ago facilitated genomic medicine, with the tumor genetic information of each patient being increasingly integrated with molecularly-guided, patient-centered, diagnosis, prognosis, and treatment ${ }^{9-11}$. With the information about tumor genomics, druggable genetic targets can be identified that can potentially offer better patient outcomes ${ }^{10}$. GBM is known to harbor numerous genetic mutations, some of which give rise to genetic instability and additional mutations, and therefore, a heterogeneous response to treatment ${ }^{12-14}$. Clear correlation of genetic changes to outcomes in IDH-wildtype GBM have not been consistently demonstrated, although some reports suggest that particular mutations drive aggressive behavior ${ }^{15-17}$.

Over the past decade, MRI-based radiomics has emerged as a promising method in providing noninvasive and quantitative biomarkers that offer insights about the phenotypic characteristics of $\mathrm{GBMs}^{18-}$ 23. Radiomics can capture coarse and subtle characteristics of the tumor and its surroundings through quantification of the features of shape, distribution of intensity, texture, or higher-level statistics and combining them with machine learning $(\mathrm{ML})$ approaches to build predictive models of a given disease endpoint, i.e. OS, recurrence, genomics, etc. ${ }^{18,24}$.

In this study, we aimed to assess the potential of integrating multi-omics prognostic characteristics, including clinical measures, radiomics, MGMT methylation, and genomics, to predict OS in GBM patients. We postulate that multi-omics data integration can capture multi-faceted tumor characteristics at different scales, i.e., molecular (genomics and MGMT methylation), macroscopic (radiomics), and clinical, and therefore provide the clinicians with a comprehensive representation of the patient's condition and risk, towards facilitating personalized treatment planning and more efficient clinical trial stratification. Here, for the first time, we explore the role of tumor genomics obtained by NGS as a prognostic method in prediction of OS, and promote a radiomic signature based on widely available preoperative conventional MRI scans by combining conventional and deep radiomics.

\section{Materials And Methods}

\section{Data Description}

This retrospective study was approved by the institutional review board of the University of Pennsylvania and was compliant with the Health Insurance Portability and Accountability Act. A waiver of informed consent was issued for this retrospective analysis but all patients had provided their informed consent at the time of their imaging. All methods were carried out in accordance with relevant guidelines and regulations. We retrospectively collected a cohort of $n=617$ adult patients, who underwent pre-operative MRI scanning followed by surgical resection at HUP, between 2006 and 2018 and were histopathologically diagnosed with de novo GBM. Patients were excluded if: 1) their conventional MRI scans were incomplete (at least one of the conventional MRI sequences was missing);2) IDH mutation was detected; 3 ) treated outside of HUP; or 4) not followed from the time of surgery until death to determine their OS. A cohort of $n=516$ patients with newly diagnosed GBM were included and divided 
between a discovery $(n=404)$ and an independent replication $(n=112)$ cohort. Table 1 summarizes the characteristics of the included patients. We collected baseline demographics (age and gender), extent of tumor resection (EOR), MGMT promoter methylation, and NGS data for all patients. All the patients had been followed at HUP until deceased.

Table 1

Characteristics of the included patients in the discovery and independent replication cohorts

\begin{tabular}{|lll|}
\hline & Discovery Cohort & Replication Cohort \\
\hline Demographics & & \\
\hline No. of Patients & 404 & 112 \\
\hline Median Age (years) & 63.9 & 65.7 \\
\hline Age Range (years) & $22.0-88.5$ & $20.7-87.6$ \\
\hline No. of Females & $176(44 \%)$ & $31(28 \%)$ \\
\hline Extent of Resection (No. of Patients) & & $57(51 \%)$ \\
\hline Near/Gross total resection & $281(69 \%)$ & $55(49 \%)$ \\
\hline Partial resection or Biopsy & $123(30 \%)$ & $42(37.5 \%)$ \\
\hline MGMT Methylation Status (No. of Patients) & & $70(62.5 \%)$ \\
\hline Methylated & $43(10 \%)$ & 0 \\
\hline Unmethylated & $80(20 \%)$ & $12.2 \pm 11.0$ \\
\hline Indeterminate or Not Available (N/A) & $281(70 \%)$ & \\
\hline Survival (months) & & \\
\hline Median \pm Std & $12.1 \pm 13.8$ & \\
\hline
\end{tabular}

\section{MRI Acquisition}

All included patients had undergone pre-operative MRI acquisition on a 3 Tesla scanner (Siemens Magnetom Tim Trio, Erlangen, Germany) using a 12-channel phased array coil. Conventional MRI sequences included pre- (T1) and post-gadolinium contrast enhanced (T1-Gd) axial high-resolution threedimensional (3D) magnetization-prepared rapid acquisition with gradient echo (MPRAGE) sequence, with Repetition Time (TR)/Echo Time (TE) $=1760 / 3.1$, Flip angle $(F A)=15^{\circ}$, Field of View $(F O V)=187 \times 250$ $\mathrm{mm}^{2}$, Slice Thickness $=1 \mathrm{~mm}$, Matrix Size $=192 \times 256 \times 192$, resolution $=0.98 \times 0.98 \times 1$; axial Turbo SpinEcho T2-weighted (T2) imaging with: TR/TE $=5340 / 85, F A=160^{\circ}, F O V=200 \times 240 \mathrm{~mm}^{2}$, Slice Thickness = $3 \mathrm{~mm}$, Matrix Size $=208 \times 256 \times 64$, resolution $=0.94 \times 0.94 \times 3$; axial Turbo Spin-Echo T2-weighted fluidattenuated inversion recovery (T2-FLAIR) with TR/TE $=9420 / 141, \mathrm{FA}=170^{\circ}, \mathrm{FOV}=180 \times 240 \mathrm{~mm}^{2}$, Slice Thickness $=3 \mathrm{~mm}$, Matrix Size $=192 \times 256 \times 60$, resolution $=0.94 \times 0.94 \times 3$. 


\section{Next-Generation Sequencing (NGS)}

The resected tumor samples for the patients in this cohort were sequenced using one of the two in-house targeted NGS panels, as described below.

\section{NGS Panel 1}

Tumor samples for a cohort of $n=193$ patients were evaluated with an in-house NGS panel. This custom AmpliSeq sequencing panel was designed targeting the CDS of 45 genes as well as IDH1 p.R132, IDH2 p.R172, and BRAF p.V600. Libraries were prepared from 10-40 ng FFPE DNA using the Ion AmpliSeq Library Kit 2.0 (ThermoFisher Scientific), multiplexed, and sequenced on an lon Torrent S5 sequencer 540 chip targeting 3.4M reads per sample for $1600 \mathrm{x}$ average depth of coverage. Primary analysis and alignment of sequence reads was performed using Torrent Suite Software version 5.8 followed by SNV and indel calling using an lon Reporter v5.10 single sample somatic variant calling workflow.

Ion Reporter variants were normalized using BCFtools followed by annotation with Annovar and filtering with BCFtools. Variants with quality scores $<30$, read depth $<100$, allele frequency $<0.05$ and strand bias $>0.7$ were filtered out, as were indels in homopolymer regions greater than $8 \mathrm{bp}$ in length. Filtering on annotations retained only splice site and exonic (excluding synonymous) variants occurring in the gnomAD exome and genome databases with population frequency $<1 \%$.

Potential sequencing artifacts were identified by manual curation of recurrent variants absent in gnomAD exomes (v2.1.1), COSMIC (v90), or CLINVAR (1/27/2020) as well as sequencing 10 HapMap samples obtained from the Coriell Institute, including NA12342 from the NIGMS Human Genetic Cell Repository, with the same panel. Variants classified as artifacts were also filtered out.

Target genes comprised: TP53, PTEN, ATRX, EGFR, VEGF, PDGFRA, PIK3CA, PIK3R1, NF1, PDL 1, CTLA4, HIF1A, MDM4, RB1, STAT1, CD70, CIC, FUBP1, CDK4, ACADL, TRIM26, SMAD1, ARID2, CDKN1B, CREBZF, DNMT3A, EPHB2, ERRFI1, FGFR2, GIGYF2, KDR, KRAS, MAP3K1, MET, NIPBL, NOTCH2, NRAS, NTRK1, PTBP1, PTPN11, SETD2, SMARCB1, TP63, WRN. The hotspots included IDH1 p.R132, IDH2 p.R172, BRAF p.V600, H3F3A p.K28, TERT (chr5:1295151-1295315). However, H3F3A were effectively not covered due to presence of pseudogene, that was ignored by variant caller, and TERT showed poor amplicon performance and excluded.

\section{NGS Panel 2}

We collected the genomic data for $n=181$ patients that had their tumors sequenced in another panel of 153 actionable and prognostic genes for sequencing solid tumors, implemented and validated at our institution for clinical assessment of the resected tumors. With the Agilent Haloplex design, the panel provides a full coverage of all included genes. A complete description of this panel and the in-house data processing bioinformatics pipeline has been previously documented ${ }^{25}$.

\section{Final Genomic Cohort}


A total of 27 genes were included in both panels and therefore, used for our integrated risk stratification approach for $\mathrm{n}=374$ patients: ARID2, ATRX, BRAF, CDKN2A, CIC, DNMT3A, EGFR, FGFR2, FUBP1, IDH1, IDH2, KDR, KRAS, MDM4, MET, NF1, NOTCH2, NTRK1, PDGFRA, PIK3CA, PIK3R1, PTEN, PTPN11, RB1, SETD2, SMARCB1, TP53. We excluded patients with mutations in IDH1 or IDH2. The final genomic cohort comprised of $\mathrm{n}=266 \mathrm{IDH}$-wildtype patients with available OS data.

\section{DNA Methylation Profiling}

Genomic DNA was extracted from tumor samples, underwent bisulfite conversion, and was then amplified with primers which target DMR2 of the MGMT (0-6-methylguanine-DNA methyltransferase, NM_002412) promoter, including 4 CpG sites (chr10:131,265,519 - 131,265,537; hg19 Assembly). The PCR product was evaluated using pyrosequencing (PyroMark Q24; Qiagen, Hilden, Germany) to determine the percent methylation across the $4 \mathrm{CpG}$ sites. A sample was called methylated if both the mean and median percentage methylation calculated over the $4 \mathrm{CpG}$ sites was $\geq 10 \%{ }^{25}$ and considered low positive when mean and median level of DNA methylation are either relatively low (i.e., above the limit of detection but below $10 \%$ ) or highly variable across the $4 \mathrm{CpG}$ sites. A not detected result was determined when the mean and median percent methylation across the $4 \mathrm{CpG}$ sites were below the limit of detection (4.5\%).

\section{Image Pre-processing}

The multi-parametric MRI (MP-MRI) volumes were preprocessed using Cancer Imaging Phenomics Toolkit open-source software (CaPTk, https://www.cbica.upenn.edu/captk) ${ }^{25,26}$. For each of the patients, the raw DICOM images were converted to NIfTI format, reoriented to the left-posterior-superior coordinate system, then rigidly co-registered and resampled to a spatial resolution of $1 \times 1 \times 1 \mathrm{~mm}^{3}$ based on the SRI atlas ${ }^{27}$. All image registrations were performed using the Greedy tool (https://github.com/pyushkevich/greedy)

28 . The images were then skull-stripped using the Brain Mask Generator (BrainMaGe) ${ }^{29}$ and corrected for intensity inhomogeneities and noise. Image intensities were scaled to the range of $(0,255)$ after removing the outlier pixels that did not fall into $99.9 \%$ percentile of the image histogram. Automatic segmentation of tumoral subregions, including the enhancing tumor (ET), necrotic tumor core (NC), and peritumoral edematous/infiltrated tissue (ED), was performed with the deep learning brain tumor segmentation module of CaPTk v.1.8.1 ${ }^{25,26}$, that is based on DeepMedic ${ }^{30}$ and revised when necessary.

\section{Radiomic Feature Extraction}

The pre-processed MP-MRI scans and segmentations of tumor subregions were passed through the feature extraction panel of CaPTk to calculate radiomic features automatically, for each of the imaging scans (T1, T1-Gd, T2, T2-FLAIR) and based on each tumoral subregion, i.e., ET, NC, ED. A total of 1032 radiomic features were extracted with feature categories of first-order intensity-based statistics, histogram, volumetric, gray-level co-occurrence matrix (GLCM), gray-level run length matrix (GLRLM), gray-level size-zone matrix (GLSZM), neighborhood gray-tone difference matrix (NGTDM) ${ }^{31}$, and Collage features ${ }^{32}$. All extracted features were normalized using z-scoring before further analyses. 


\section{Deep Radiomic Features}

We further extracted a set of deep radiomic features obtained from a pre-trained deep learning model using transfer learning, i.e., VGG-19 with 16 convolutional neural networks including rectified linear unit (RELU) pooled with three fully connected layers. This model has been pre-trained on a natural image dataset ImageNet (http://www.image-net.org) and shown excellent performance in image classification tasks ${ }^{33,34}$. The architecture of VGG-19 is illustrated in Fig. 1. We selected a bounding box (or a patch) encompassing the entire tumor, i.e., the union of ET, NC, and ED, on MP-MRI (T1, T1-Gd, T2, T2-FLAIR) scans, which were then resized to a dimension of $224 \times 224$ using cubic interpolation and fed as inputs to the deep networks. Front propagation with pre-trained weights was performed for initialization and extracted 8192 features from the first two fully connected layers.

\section{Radiomic Analysis}

For radiomics analysis, as illustrated in Fig. 1, on the conventional and deep radiomic features extracted from the training cohort $(n=404)$, we applied support vector machine (SVM) classification with recursive feature elimination (RFE) for feature selection to build two classifiers (a dual classifier) in Python 3.6.5. One classifier differentiates the high-risk patients, who survived less than 6 months, from the rest of the subjects, and the second classifier discriminates the lower-risk patients, who survived beyond 18 months, from the remaining patients. After normalizing the features using z-scoring (subtraction of the mean and division with the standard deviation), we removed all features with small variations as determined by the mean absolute deviation (MAD). The adopted RFE-SVM method searches for the optimal feature subset by fitting the SVM model to the feature space and removing the weakest features until the best performing feature subset is achieved. The classifiers were trained with 5 -fold nested cross validation (nested-CV) to ensure generalizability. For nested-CV, the data were divided into five folds (for external cross-validation), and each external fold was further divided into another five folds (for internal crossvalidation). In the internal folds, feature selection and SVM hyperparameter tuning were performed. In each external fold, with the feature subset and hyperparameter values that were selected in the internal folds, SVMs were trained based on four out of five folds of the data and validated on the remaining fold. From the five SVM models trained in the external folds, the one with the best validation accuracy was chosen to be applied to the independent replication data.

The distances of the data points to the SVM hyperplanes were derived, a sigmoid function was fitted to the distances, generating two indices for the dual classifier representing the pseudo-probabilities for the data. These indices were then averaged to create a survival prediction index ( $\mathrm{SPI}_{\text {radiomics }}$ ), with a higher value indicating a higher OS (i.e., a lower-risk), and a lower value was associated with a lower OS (i.e., a higher-risk). The resulting model, with the hyperparameter values optimized through 5 -fold nested $\mathrm{CV}$ based on the training cohort $(n=404)$, was tested on the selected features in the model calculated for our independent replication cohort $(n=112)$ and SPI $I_{\text {radiomics }}$ values were generated for the patients in this cohort. 
We designed our radiomics predictive model to be compatible with clinical scenarios, where short survivor/higher risk patients may be prescribed with a supra-total resection for their surgery and dose escalation in radiation therapy. On the contrary, long-survivor/lower risk patients may receive aggressive local treatments without supra-total resection ${ }^{21}$. Therefore, we have regulated our predictive model to characterize the two extremes that can affect the clinical decision making about the appropriate management recommendation of these patients.

\section{Risk Assessment}

To relate these features with overall survival, we employed six Cox proportional hazards (Cox-PH) models. We trained Cox proportional hazards models for different combinations of predictors. Models were fit with the following different levels of data integration:

(1) Clinical measures, including demographics (age, gender), and extent of resection (EOR).

1. Clinical measures and $M G M T$ methylation status.

2. Clinical measures and radiomics (i.e., SPI radiomics $_{\text {) }}$.

3. Clinical measures, MGMT methylation, and radiomics.

4. Clinical measures, MGMT methylation, and genomics.

5. Clinical measures, MGMT methylation, radiomics, and genomics.

The multi-omics risk assessment method is indicated in Fig. 2.

For the genomic variables (for $\mathrm{n}=112$ patients), we considered a Cox Proportional Hazards (Cox-PH) model with least absolute shrinkage and selection operator (LASSO) penalty, to reduce the dimensionality of the genomic information by identifying a subset of variables that are predictive of OS ${ }^{35}$. The LASSO estimator performs feature selection by shrinking the number of regression coefficients to zero. This degree of shrinkage is controlled by the parameter $\lambda$, which is typically found through CV as the value which maximizes the partial likelihood. Because the random partitions in CV can produce highly variable results, both in terms of the selected variables and the model performance, we used two nested CV loops 36: the inner loop selected the optimal $\lambda$, and the outer loop assessed out-of-sample performance. In each outer loop, the data was randomly partitioned into outer training $(n=75)$ and outer test $(n=37)$ sets. In the inner loop, 3-fold CV on the outer training set was repeated 100 times to determine the value of $\lambda_{\min }$ that minimized average deviance over the repetitions. Prediction performance of the model fit using $\lambda_{\min }$ was then assessed on the outer test set. The outer loop was repeated 100 times and the value of $\lambda_{\text {final }}$ with highest c-index in the testing sets was selected as the parameter in the final LASSO regression.

Each of the 6 models were fit on the same set of subjects $(n=112)$ that had complete clinical, MGMT methylation, radiomic, and genomics data. We considered maximum partial likelihood estimator (MPLE), as an estimator of the regression coefficients for variables in the models. For all models, prediction accuracy was assessed using the concordance (c-) index, which ranges from 0 (worse fit) to 1 (better fit). We further gauged model performance based on the Integrated Brier Score (IBS) ranging from 0 (better) 
to 1 (worse) as compared to a reference model. Statistical analyses were performed in $\mathrm{R}$ version 3.6.0 ( $\mathrm{R}$ Foundation for Statistical Computing, Vienna, Austria). There was no censoring, as the event time was observed for all individuals. This study did not have any missing data.

\section{Results}

\section{Radiomics Signature of Overall Survival}

Radiomic analysis demonstrated an AUC of 0.78 (95\% Cl: $0.70-0.85)$ for the high-risk (6-month) and 0.75 (95\% Cl: 0.65-0.84) for the low-risk (18-month) classifier, in the training cohort. In the independent replication cohort, the classification performance of high-risk and low-risk classifiers in terms of AUC were 0.75 (95\% Cl: 0.64-0.79) and 0.63 (95\% Cl: 0.52-0.71), respectively. Figure 3 (A) presents box plots of $\mathrm{SPI}_{\text {radiomics }}$ vs actual survival in months (greater value of $\mathrm{SPI}_{\text {radiomics }}$ predicts more prolonged survival, and lower SPI predicts shorter survival) for the independent and training cohort. A summary of top ranked radiomic features for the high and low-risk classifiers can be found in Fig. 3 (B), suggesting that the majority of selected features are among deep features.

\section{Risk Stratification}

The Cox-PH model using clinical variables (i.e., age, gender, and EOR), showed a c-index of 0.65 (95\% Cl: $0.60-0.70)$ with the largest coefficient for EOR with HR $=0.43$ (95\% Cl: $0.29-0.64$; $p$-value < 0.0001$)$, indicating that gross total or near total resection of the tumor was associated with longer OS. Adding MGMT methylation to the clinical variables slightly improved the c-index of the created Cox model to 0.67 (0.62-0.72). In this model, the significant predictors were MGMT methylation with $\mathrm{HR}=0.56(95 \% \mathrm{Cl}$ : $0.36-0.87$; p-value $=0.01)$, and EOR with HR $=0.44(95 \% \mathrm{Cl}: 0.29-0.65 ; \mathrm{p}$-value< 0.0001$)$. These results suggest that MGMT-methylated tumors and larger extent of resection are associated with a lower hazard of short survival.

The model including clinical and survival prediction index (SPI radiomics $)$ covariates returned an improved c-index $=0.70$ (95\% Cl: 0.65-0.75), compared to the clinical model. This model showed the largest negative coefficient for $\mathrm{SPI}_{\text {radiomics }}$ with $\mathrm{HR}=0.13$ (95\% Cl: 0.04-0.45; $\mathrm{p}$-value = 0.001), implying that the higher $\mathrm{SPI}_{\text {radiomics }}$ was associated with lower hazard of short survival. After adding MGMT methylation status to the clinical and $\mathrm{SPI}_{\text {radiomics }}$ variables, c-index improved to 0.72 (95\% Cl: $\left.0.68-0.77\right)$. SPI $\mathrm{I}_{\text {radiomics }}$ showed a low HR of 0.07 (95\% Cl: 0.02-0.27; p-value = 0.0001).

The genomic variables were penalized using LASSO method in a Cox-PH model including genomic mutations and the selected genes were EGFR, MET, NOTCH2, PDGFRA, and RB1. The Cox-PH model including clinical, MGMT methylation status, and genomic variables showed a c-index of $0.70(95 \% \mathrm{Cl}$ : 0.66-0.75), performing similarly to the model composed of clinical and SPI $\mathrm{radiomics}_{\text {covariates. }}$

Significant predictors of OS in this model were patient's EOR (HR $=0.35,95 \% \mathrm{Cl}: 0.22-0.54$; $\mathrm{p}$-value < 0.0001), Age $(H R=1.03,95 \%$ Cl: 1.01-1.05; $p$-value = 0.002), MGMT methylation status $(H R=0.53,95 \%$ 
Cl: $0.34-0.81 ; \mathrm{p}$-value $=0.004), R B 1(\mathrm{HR}=0.38,95 \% \mathrm{Cl}: 0.19-0.76 ; \mathrm{p}$-value $=0.006)$, and NOTCH2 $(\mathrm{HR}=$ $0.2,95 \% \mathrm{Cl}: 0.06-0.71 ; \mathrm{p}$-value $=0.01)$.

Finally, the last model integrating all data sources, i.e., clinical, $M G M T$ methylation, $\mathrm{SPI}_{\text {radiomics, }}$ and genomic, showed an improved c-index $=0.75$ (95\% Cl: 0.71-0.78), where significant covariates included EOR (HR = 0.32, 95\% Cl: 0.20-0. 50; $\mathrm{p}$-value < 0.0001), SPI radiomics $(\mathrm{HR}=0.06,95 \% \mathrm{Cl}: 0.02-0.23$; $\mathrm{p}$-value < $0.0001), M G M T$ methylation status ( $\mathrm{HR}=0.42,95 \% \mathrm{Cl}: 0.27-0.67$; $\mathrm{p}$-value $=0.0003)$, Age $(\mathrm{HR}=1.03,95 \%$ Cl: 1.01-1.04; p-value =0.004), NOTCH2 (HR=0.15, 95\% Cl: 0.04-0.55; p-value $=0.004)$, and $R B 1(\mathrm{HR}=$ $0.42,95 \% \mathrm{Cl}: 0.21-0.83 ; \mathrm{p}$-value $=0.01)$. Together, these results suggest that our fully integrated (i.e., the multi-omics) model containing all derived features is the most predictive of survival time. A forest plots of the covariates in each of the models and their hazard ratios is provided in Fig. 4.

Table 2 summarizes the performances of the 6 designed Cox-PH models. The results in this table show an increased c-index and reduction in IBS error, suggesting an improvement in risk stratification when a further layer of information is added to the integrated prognostic models. Including MGMT methylation with the clinical variables yielded a $14.2 \%$ reduction in prediction error with respect to the reference IBS, integrating radiomics with clinical data resulted in $15 \%$ decrease, a combination of clinical, MGMT methylation, and radiomics achieved $19.4 \%$ decrease in prediction error, similar to an integration of clinical, MGMT methylation, and genetics with $19.8 \%$ decline in IBS. The highest performance was achieved with the model composed of clinical, MGMT methylation, radiomics, and genomics, as evidenced by the c-index of 0.75 and IBS reduction of $24.8 \%$. 
Table 2

Performance metrics for the Cox-PH models

\begin{tabular}{|c|c|c|c|}
\hline Model & $\begin{array}{l}\text { c-index }(95 \% \\
\left.\mathrm{Cl}^{+}\right)\end{array}$ & IBS & $\begin{array}{l}\text { IBS } \\
\text { Difference* }\end{array}$ \\
\hline Model 1: Clinical & $0.65(0.6,0.7)$ & 0.101 & $-10.3 \%$ \\
\hline Model 2: Clinical + MGMT methylation & $\begin{array}{l}0.67(0.62 \\
0.72)\end{array}$ & 0.097 & $-14.2 \%$ \\
\hline Model 3: Clinical + Radiomics & $\begin{array}{l}0.70(0.65, \\
0.75)\end{array}$ & 0.096 & $-15 \%$ \\
\hline Model 4: Clinical + MGMT methylation + Radiomics & $\begin{array}{l}0.72(0.68, \\
0.77)\end{array}$ & 0.091 & $-19.4 \%$ \\
\hline Model 5: Clinical + MGMT methylation + Genomics & $\begin{array}{l}0.70(0.66 \\
0.75)\end{array}$ & 0.091 & $-20.3 \%$ \\
\hline $\begin{array}{l}\text { Model 6: Clinical + MGMT methylation + Radiomics + } \\
\text { Genomics }\end{array}$ & $\begin{array}{l}0.75(0.72 \\
0.79)\end{array}$ & 0.086 & $-24.8 \%$ \\
\hline \multicolumn{4}{|l|}{${ }^{\dagger} \mathrm{Cl}$ : Confidence Interval } \\
\hline
\end{tabular}

Survival curves for high, medium, and low risk groups are depicted in Fig. 5, suggesting a synergistic interaction of different sources of information in stratification of high, medium, and low risk patients. As apparent from the survival curves, with the addition of a layer of information, discrimination of the risk groups improves. Model 2 with a combination of clinical and MGMT methylation data can better differentiate the low from medium risk groups compared with model 1 with only clinical information. The incremental value of radiomics to this combination can be observed from the curves in model 4 , where the three risk groups are distinctly stratified. Genomics build on this discriminative potential of clinical, $M G M T$, and radiomics variables in model 6 and the risk groups become further differentiated. The results for model 3, i.e., clinical and radiomics, show that in absence of any sequencing, including MGMT methylation and genomics, radiomics signature created based on pre-operative MRI, can provide an added value to the clinical predictors of OS and provide a distinct stratification of the risk groups. The similarity in performance of models 4 and 5 (as suggested by their c-index of 0.70 in Table 2) is further evidenced from their corresponding survival curves.

\section{Discussion}

This study introduces a multivariate integration of multi-omics data for risk stratification of patients with newly diagnosed, treatment-naïve IDH-wildtype GBM. We used information collected from several sources of data to build multiple models, each with different layers of prognostic information for disease stratification. Starting from the base model including the least available information for the patients with 
GBM, i.e., clinical variables of age, gender, and extent of resection, we showed incremental value of radiomics, MGMT methylation, and genomic data obtained by NGS sequencing of the tumor samples, resulting in a multi-omics model with superior performance.

Despite marked variation in treatment responsiveness and outcomes, nearly all patients with newly diagnosed GBM still receive the same standard radiation and temozolomide-based therapy, underscoring a lack of personalized treatment in GBM relative to many other solid tumors ${ }^{37}$. Moreover, interpatient heterogenetiy in both tumor biology and clinical characteristics has made it difficult to accurately determine the efficacy of experimental treatments, particularly in newly diagnosed GBM trials with progression-free survival (PFS) or OS endpoints ${ }^{38,39}$. Our findings suggest that multi-omics or multi-scale fusion of data can provide a comprehensive portrayal of tumor biology and the patient's likely response to the treatment. If validated in a larger, multi-center study, this survival prediction tool has the potential for widespread clinical and research use, allowing for more personalized treatment in routine practice and more efficient clinical trial design in the newly diagnosed setting.

In the present study, the image-based predictive model of OS builds upon and reinforces prior studies ${ }^{20,23}$ by employing an advanced computational methodology based on high-throughput data, i.e., conventional and deep radiomic features. We showed generalizability of our non-invasive prognostic approach to unseen data, evidenced by the classification performance of our ML models. An important strength of our proposed signature is that it is derived from pre-operative conventional MRI scans which are acquired as a part of standard-of-care diagnosis of patients with GBM. In both of our ML classifiers, i.e., high-risk and low-risk models, most of the selected features were among deep features derived from T2 and T2-FLAIR scans, emphasizing the significance of these features in quantifying higher-order patterns. GBM tumors exhibit spatial heterogeneity arising from variation in cellular density, vascularization, and necrosis across their area, and deep learning features enable detection of phenotypic characteristics within preoperative images that may not be captured comprehensively by only conventional radiomic features.

The survival prediction index ( $\left.\mathrm{SPI}_{\text {radiomics }}\right)$ was used to fuse with other omics data for risk stratification of patients with GBM. As the results suggest, $\mathrm{SPI}$ radiomics provided additive value over the clinical and MGMT data for risk assessment, improved the concordance index, and provided more distinctive separation of the low, medium, and high-risk groups. Genetics further improved risk stratification in synergy with other omics. Added value of a radiomics signature to clinical and MGMT methylation in prediction of OS and PFS in patients with GBM has been reported in only a few studies with smaller cohorts 22,40 . The current study contributes to the improvement of the interpretability, predictive applicability and computational efficiency of existing machine learning algorithms, thereby increasing the precision of population-based registries and validate current and future prediction tools.

In studies including only genomics, genetic classification of IDH-wildtype GBM ${ }^{15}$ has not yielded prognostic information, although a recent transcriptomic classification based on developmental and metabolic axes has dissected apart subclasses of GBM with prognostic significance and contrasting metabolic vulnerabilities ${ }^{41}$. Our approach revealed two genes, RB1 (retinoblastoma tumor suppressor 
protein) and $\mathrm{NOTCH} 2$, previously studied for their role in oncogenesis and, and as potential targets for glioma therapy. RB1 was previously discovered to be a tumor suppressor gene, like TP53; inactivation of $R B 1$ in preclinical models suggested it plays a significant role in carcinogenesis ${ }^{42}$. $R B 1$ is involved in the cell cycling pathway, allowing progression from $\mathrm{G} 1$ to $S$ phase, activated by phosphorylation and regulated by $C D K 4 / C K 6$ protein kinases ${ }^{43}$. In a study of $C D K 4 / 6$ inhibitors in xenografts, $R B 1$ was identified as a determinant of therapeutic efficacy ${ }^{44}$. Loss of RB1 transcript expression was seen in $10.6 \%$ of GBMs in the TCGA analysis, and these tumors were highly enriched for the proneural subtype; loss of $R B 1$ transcript expression was not associated with a difference in overall survival on univariate analysis ${ }^{44}$. To date, a specific inhibitor of the $R B 1$ signaling pathway has not been developed; however, palbociclib, an inhibitor of the $C D 4 / C D K$ protein kinase pathways, was reported to be ineffective in a phase 2 trial against recurrent, $R B 1$ positive, recurrent GBM ${ }^{43}$.

Notch signaling, discovered in Drosophila nearly a century ago, is known to be involved in numerous cellular processes, including signaling in solid tumors ${ }^{45}$. Notch signaling has also been implicated in angiogenesis as well as cancer drug chemo-resistance and radioresistance of cancer stem cells; a role for Notch is well established in hematological malignancies, but in solid tumors, its role is highly contextual: an oncogene is some cancers but a tumor suppressor in others ${ }^{45}$. Notch signaling occurs at the interface of the tumor-stromal microenvironment ${ }^{46}$. NOTCH2 is a positive regulator of transcription ${ }^{47}$; it is found as a somatic gene mutation in a small percentage $(23 \%)$ of pleomorphic xanthoastrocytomas, along with other somatic mutations (BRAF V600E, NF1, etc.), and could be involved in the pathogenesis of PXAs 47 or in anaplastic astrocytomas, where it was found in $31 \%$ of these tumors ${ }^{48}$.

This paper presents several contributions. First, it presents a comprehensive study of prognosis of OS leveraging machine learning-based integration of clinical, imaging, and genomic data drawing from a cohort totaling 516 patients, including an independent replication dataset. To our knowledge, this is the first study of this size to explore the synergistic value of genetic, imaging and clinical predictive factors. Second, it demonstrated that a combination of deep learning and conventional radiomics produces a strong predictive panel of radiomic features for OS using standard, routinely acquired MRI scans. Third, it demonstrates the relative value of each omics in the context of additive contribution of clinical, imaging, and genetic variables. Critically, proper integration of all these measures via machine learning produced high predictive value on an independent test cohort of 112 patients, thereby further bolstering our confidence in the reproducibility and clinical value of these emerging Al-based integrated precision diagnostic indices.

A limitation of our study is the single institutional data analysis. To ac hieve a generalizable method, it would be beneficial to explore data collected from multiple institutions. Furthermore, the sample size for risk stratification ( $n=112$ ) was a potential limitation, which was mainly due to the unavailability of all omics information for all patients in our cohort. In particular, as broader molecular datasets become available on more patients, the prognostic implications of the molecular changes in the context of other 
omics may become clearer. Future studies would benefit from multi-institutional, prospective, and larger study population, a goal which the ReSPOND consortium is aiming to achieve ${ }^{49}$.

In conclusion, the present study fuses multiple omics data, namely clinical information, MGMT methylation, radiomics, and genetics, to accurately model clinical outcome in patients with newly diagnosed GBM. Accurate stratification of risk groups may facilitate improved patient management through personal optimization of treatment decisions, as well as effective risk stratification of patients for newly diagnosed GBM clinical trials.

\section{Declarations}

\section{Authors' Contributions:}

Conception and design: A Fathi Kazerooni, S Saxena, M Nasrallah, C Davatzikos

Development of methodology: A Fathi Kazerooni, S Saxena, D Tu, V Bashyam, R Shinohara, E Toorens, T Ganguly, I Verginadis, S Bakas, M Nasrallah, C Davatzikos

Acquisition of data: E Mamourian, C Sako, E Toorens, T Ganguly, S Bakas

Preprocessing of data: C Sako, E Mamourian, A Fathi Kazerooni, E Toorens

Analysis and interpretation of data (e.g., statistical analysis, biostatistics, computational analysis): A Fathi Kazerooni, S Saxena, D Tu, H Akbari, V Bashyam, R Shinohara

Writing, review, and/or revision of the manuscript: A Fathi Kazerooni, S Saxena, E Toorens, D Tu, V Bashyam, H Akbari, E Mamourian, C Sako, C Koumenis, I Verginadis, R Verma, R Shinohara, A Desai, R Lustig, S Brem, S Mohan, S Bagley, T Ganguly, D O'Rourke, S Bakas, M Nasrallah, C Davatzikos

\section{References}

1. Stupp, R. et al. Effect of tumor-treating fields plus maintenance temozolomide vs maintenance temozolomide alone on survival in patients with glioblastoma a randomized clinical trial. JAMA - $J$ Am Med Assoc, 318 (23), 2306-2316 (2017).

2. Helseth, R. et al. Overall survival, prognostic factors, and repeated surgery in a consecutive series of 516 patients with glioblastoma multiforme. Acta Neurol Scand, 122 (3), 159-167 (2010).

3. Ellingson, B. M. et al. Validation of postoperative residual contrast-enhancing tumor volume as an independent prognostic factor for overall survival in newly diagnosed glioblastoma. Neuro Oncol, 20 (9), 1240-1250 (2018).

4. Weller, M. et al. Review European Association for Neuro-Oncology (EANO) guideline on the diagnosis and treatment of adult astrocytic and oligodendroglial gliomas. Lancet Oncol, 18 (6), e315-e329 (2017). 
5. Weller, M. et al. MGMT promoter methylation in malignant gliomas: ready for personalized medicine? Nat Rev Neurol, 6 (1), 39-51 (2010).

6. Maier-Hein, L. et al. BIAS: Transparent reporting of biomedical image analysis challenges. Med Image Anal, 66, 101796 (2020).

7. Malmström, A. et al. Neuro-Oncology Practice glioma ? Results of an international survey regarding use of MGMT analyses for glioma. 2020;7(September 2019):68-76.

8. Hegi, M. E. et al. MGMT Promoter Methylation Cutoff with Safety Margin for Selecting Glioblastoma Patients into Trials Omitting Temozolomide: A Pooled Analysis of Four Clinical Trials. Published online 2019:1809-1817.

9. Kulski, J. K. Next-generation sequencing-an overview of the history, tools, and "omic" applications. Next Gener Seq App/ challenges. Published online2016:3-60.

10. Hyman, D. M., Taylor, B. S. \& Baselga, J. Implementing Genome-Driven Oncology., 168 (4), 584-599 (2017).

11. Fathi Kazerooni, A., Bakas, S., Saligheh Rad, H. \& Davatzikos, C. Imaging signatures of glioblastoma molecular characteristics: A radiogenomics review.J Magn Reson Imaging. 2020;52(1).

12. Brennan, C. W. et al. The somatic genomic landscape of glioblastoma., 155 (2), 462 (2013).

13. Belden, C. J. et al. Genetics of glioblastoma: A window into its imaging and histopathologic variability., 31 (6), 1717-1740 (2011).

14. Cohen, A. et al. DNA copy number analysis of Grade II-III and Grade IV gliomas reveals differences in molecular ontogeny including chromothripsis associated with IDH mutation status. Acta Neuropathol Commun, 3, 34 (2015).

15. Verhaak, R. G. W. et al. Integrated genomic analysis identifies clinically relevant subtypes of glioblastoma characterized by abnormalities in PDGFRA, IDH1, EGFR, and NF1., 17 (1), 98-110 (2010).

16. Barthel, F. P. et al. Longitudinal molecular trajectories of diffuse glioma in adults. Nature, $\mathbf{5 7 6}$ (7785), 112-120 (2019).

17. Binder, Z. A. et al. Epidermal growth factor receptor extracellular domain mutations in glioblastoma present opportunities for clinical imaging and therapeutic development., 34 (1), 163-177 (2018).

18. Gillies, R. J., Kinahan, P. E., Hricak, H. \& Radiomics Images are more than pictures, they are data., 278 (2), 563-577 (2016).

19. Bae, S. et al. Radiomic MRI phenotyping of glioblastoma: Improving survival prediction., 289 (3), 797-806 (2018).

20. Macyszyn, L. et al. Imaging patterns predict patient survival and molecular subtype in glioblastoma via machine learning techniques. Neuro Oncol, 18 (3), 417-425 (2016).

21. Fathi Kazerooni, A. et al. Cancer Imaging Phenomics via CaPTk: Multi-Institutional Prediction of Progression-Free Survival and Pattern of Recurrence in Glioblastoma.JCO Clin Cancer Informatics. 2020;(4):234-244. 
22. Kickingereder, P. et al. Radiomic subtyping improves disease stratification beyond key molecular, clinical, and standard imaging characteristics in patients with glioblastoma. Neuro Oncol, 20 (6), 848-857 (2018).

23. Bakas, S., Shukla, G., Akbari, H. \& Erus, G. Overall survival prediction in glioblastoma patients using structural magnetic resonance imaging (MRI): advanced radiomic features may compensate for lack of advanced MRI modalities. 2020;7(3):1-18.

24. Kazerooni, A. F. \& Davatzikos, C.. Computational Diagnostics of GBM Tumors in the Era of Radiomics and Radiogenomics BT - Brainlesion: Glioma, Multiple Sclerosis, Stroke and Traumatic Brain Injuries. In: Crimi A, Bakas S, eds.Springer International Publishing; 2021:30-38.

25. Davatzikos, C. et al. Cancer imaging phenomics toolkit: quantitative imaging analytics for precision diagnostics and predictive modeling of clinical outcome Cancer imaging phenomics toolkit : quantitative imaging analytics for precision diagnostics and. 2020;5(1).

26. Pati, S., Singh, A. \& Rathore, S. et al. The cancer imaging phenomics toolkit (captk): Technical overview. In: International MICCAI Brainlesion Workshop.Springer; 2019:380-394.

27. Rohlfing, T., Zahr, N. M., Sullivan, E. V. \& Pfefferbaum, A. The SRI24 multichannel atlas of normal adult human brain structure. Hum Brain Mapp, 31 (5), 798-819 (2010).

28. Yushkevich, P. A. et al. Fast automatic segmentation of hippocampal subfields and medial temporal lobe subregions in 3 Tesla and 7 Tesla T2-weighted MRI. Alzheimer's Dement, 7 (12), 126-127 (2016).

29. Thakur, S. et al. Brain extraction on MRI scans in presence of diffuse glioma: Multi-institutional performance evaluation of deep learning methods and robust modality-agnostic training., 220, 117081 (2020).

30. Kamnitsas, K. et al. Efficient multi-scale 3D CNN with fully connected CRF for accurate brain lesion segmentation. Med Image Anal, 36, 61-78 (2017).

31. Zwanenburg, A. et al. The image biomarker standardization initiative: standardized quantitative radiomics for high-throughput image-based phenotyping., 295 (2), 328-338 (2020).

32. Prasanna, P., Tiwari, P. \& Madabhushi, A. Co-occurrence of local anisotropic gradient orientations (CoLIAGe): a new radiomics descriptor. Sci Rep, 6 (1), 1-14 (2016).

33. Simonyan, K. \& Zisserman, A. Very deep convolutional networks for large-scale image recognition. arXiv Prepr arXiv14091556. Published online 2014.

34. Krizhevsky, A., Sutskever, I. \& Hinton, G. E. Imagenet classification with deep convolutional neural networks. Adv Neural Inf Process Syst, 25, 1097-1105 (2012).

35. Simon, N., Friedman, J. H., Hastie, T. \& Tibshirani, R. Regularization Paths for Cox's Proportional Hazards Model via Coordinate Descent.J Stat Software;Vol 1, Issue 5. Published online March 9 , 2011.

36. Varma, S. \& Simon, R. Bias in error estimation when using cross-validation for model selection. BMC Bioinformatics, 7 (1), 91 (2006). 
37. White, K. et al. New hints towards a precision medicine strategy for IDH wild-type glioblastoma. Ann Oncol, 31 (12), 1679-1692 (2020).

38. Ventz, S. et al. Design and Evaluation of an External Control Arm Using Prior Clinical Trials and RealWorld Data. Clin Cancer Res, 25 (16), 4993 LP - 5001. (2019).

39. Taylor, J. W., Molinaro, A. M., Butowski, N. \& Prados, M. Clinical trial endpoints for patients with gliomas. Neuro-Oncology Pract, 4 (4), 201-208 (2017).

40. Senders, J. T. et al. An online calculator for the prediction of survival in glioblastoma patients using classical statistics and machine learning. Neurosurgery, 86 (2), E184-E192 (2020).

41. Garofano, L. et al. Pathway-based classification of glioblastoma uncovers a mitochondrial subtype with therapeutic vulnerabilities. Nat cancer, 2 (2), 141-156 (2021).

42. Wiseman, R. W., Cochran, C., Dietrich, W., Lander, E. S. \& Söderkvist, P. Allelotyping of butadieneinduced lung and mammary adenocarcinomas of B6C3F1 mice: frequent losses of heterozygosity in regions homologous to human tumor-suppressor genes. Proc Natl Acad Sci, 91 (9), 3759-3763 (1994).

43. Taylor, J. W. et al. Phase-2 trial of palbociclib in adult patients with recurrent RB1-positive glioblastoma. J Neurooncol, 140 (2), 477-483 (2018).

44. Goldhoff, P. et al. Clinical stratification of glioblastoma based on alterations in retinoblastoma tumor suppressor protein (RB1) and association with the proneural subtype. J Neuropathol Exp Neurol, 71 (1), 83-89 (2012).

45. Ranganathan, P., Weaver, K. L. \& Capobianco, A. J. Notch signalling in solid tumours: a little bit of everything but not all the time. Nat Rev Cancer, 11 (5), 338-351 (2011).

46. Egloff, A. M. \& Grandis, J. R. Molecular pathways: context-dependent approaches to Notch targeting as cancer therapy. Clin cancer Res, 18 (19), 5188-5195 (2012).

47. Zou, H. et al. Molecular features of pleomorphic xanthoastrocytoma. Hum Pathol, 86, 38-48 (2019).

48. Killela, P. J. et al. The genetic landscape of anaplastic astrocytoma. Oncotarget, 5 (6), 1452 (2014).

49. Davatzikos, C. et al. Al-based prognostic imaging biomarkers for precision neuro-oncology: the ReSPOND consortium. Neuro Oncol, 22 (6), 886-888 (2020).

\section{Figures}




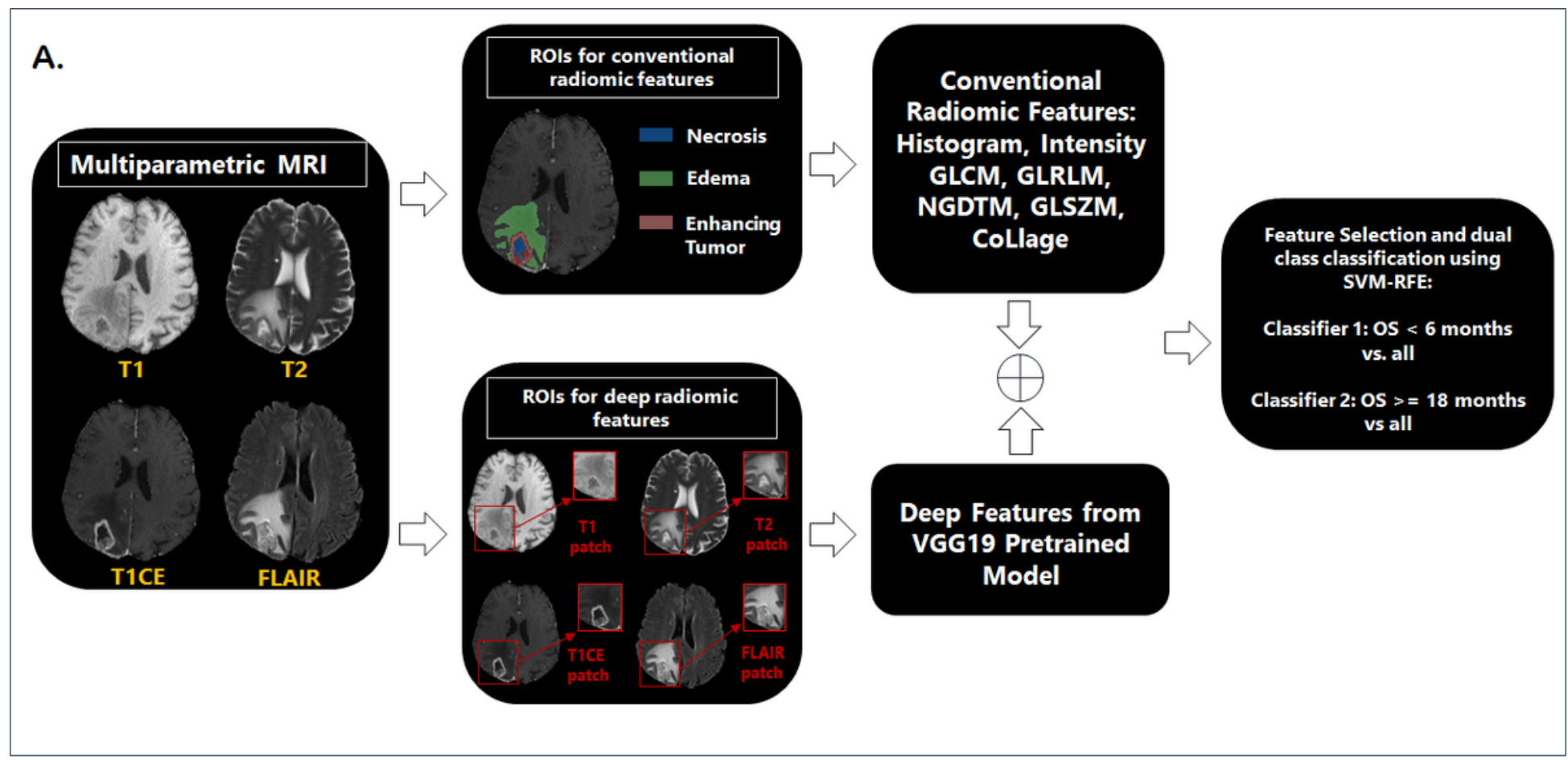

B.

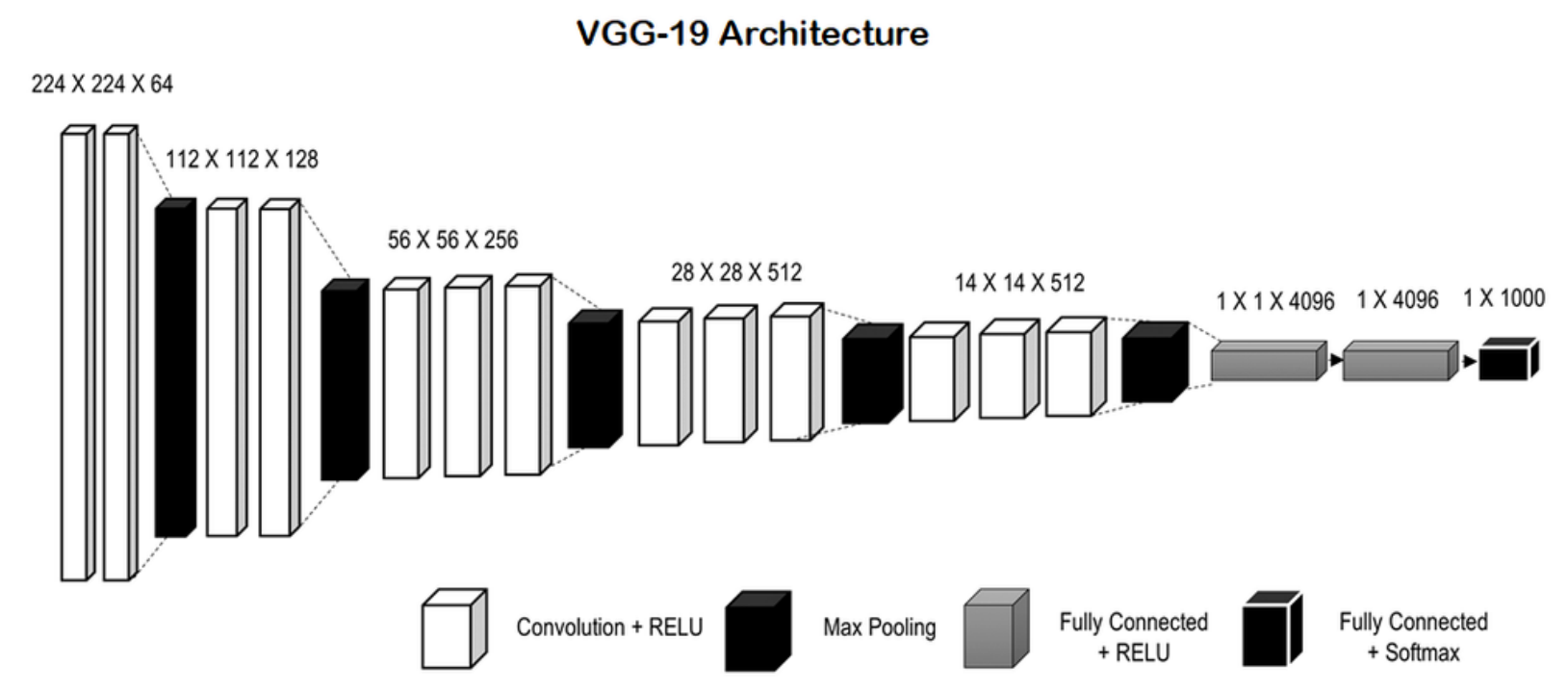

Figure 1

(A) schematic illustration of Radiomic analysis pipeline; (B) The architecture of VGG-19 network used for extraction of deep radiomic features. 
Radiomics

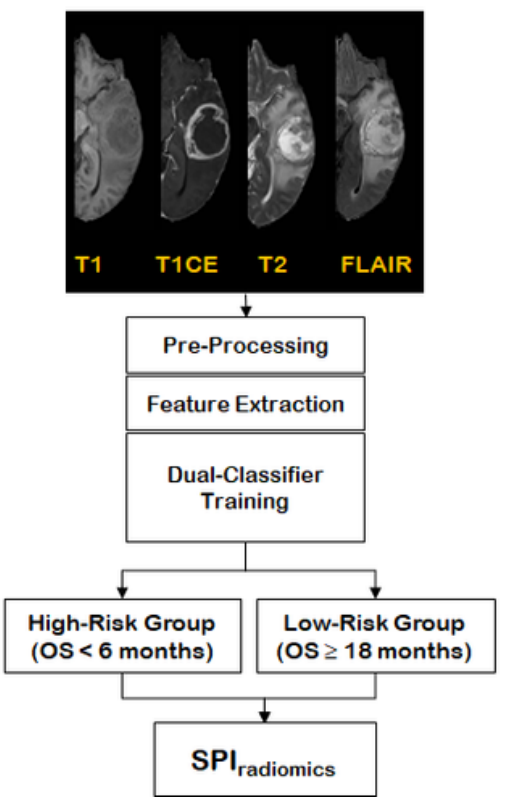

Clinical

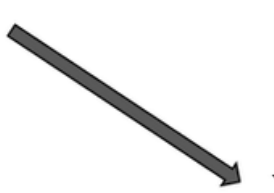

Risk Stratification
Molecular

(MGMT Methylation, genomics)
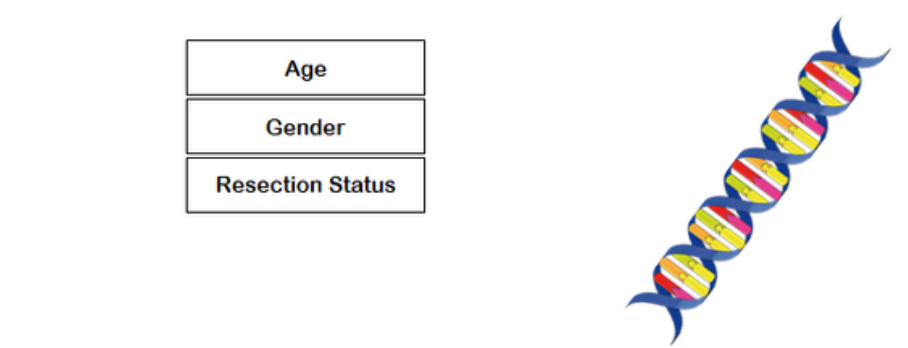

\section{Figure 2}

Multi-omic analysis method for risk stratification of patients with IDH-wildtype GBM tumors based on their radiomic signature (SPIradiomics), Clinical characteristics (age, gender, and extent of resection (EOR)), and molecular information (MGMT methylation and genomics, obtained by next-generation sequencing (NGS) of the tumor samples). 


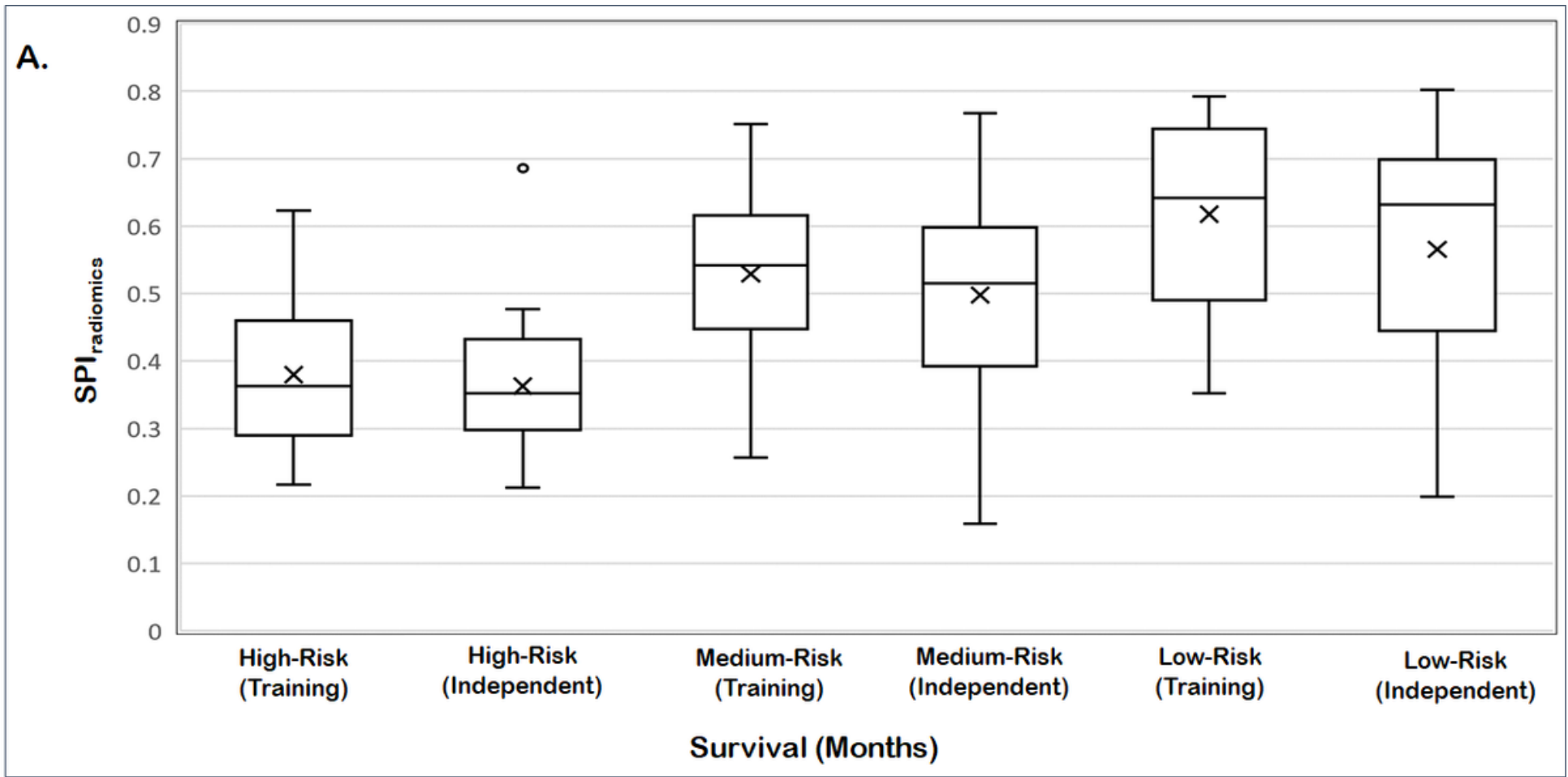

B.
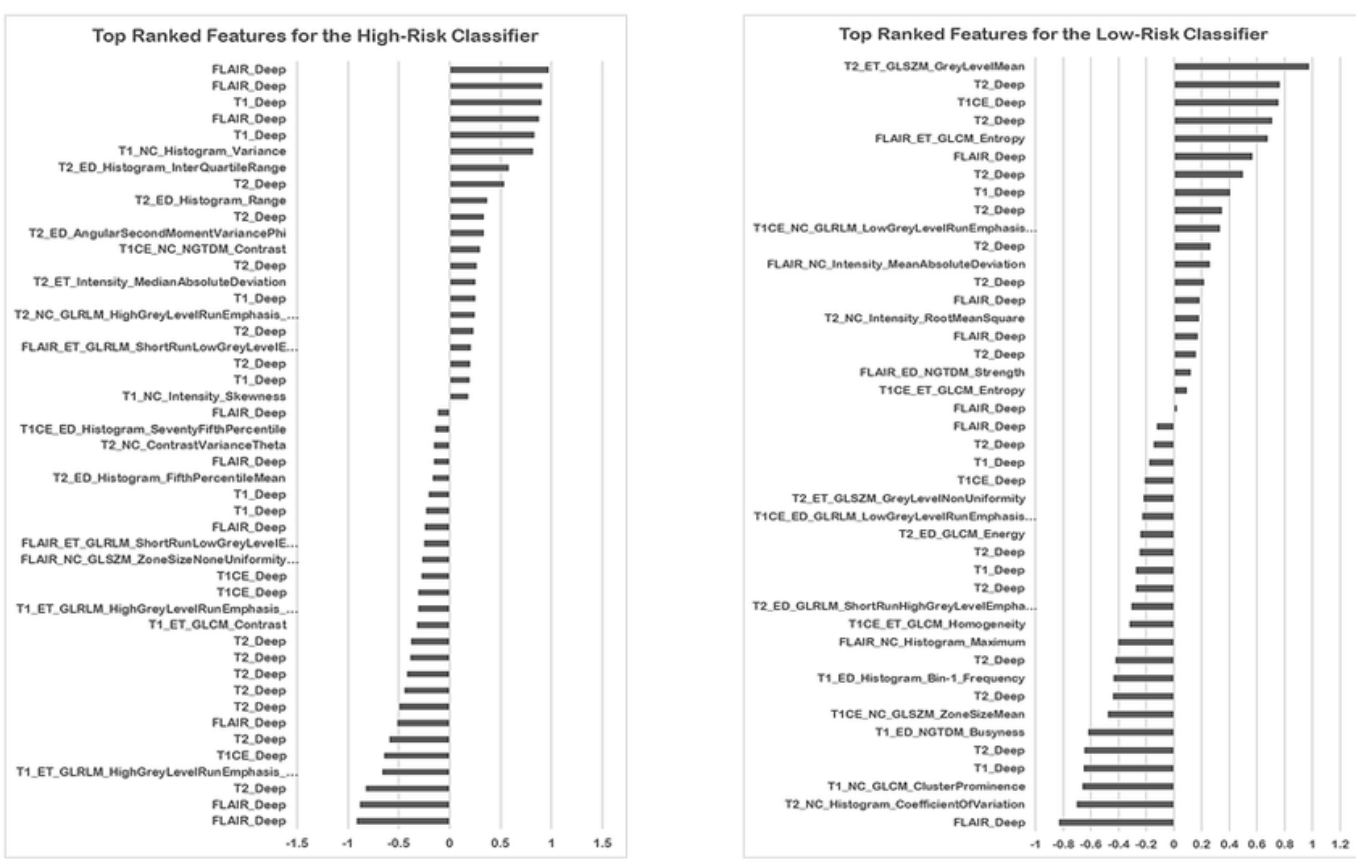

Figure 3

(A) SPIradiomics versus survival for high-risk ( $<6$ months), medium-risk ( $\geq 6,<18$ months), and low-risk ( $\geq 18$ months) patient groups for the training and independent cohorts. Survival was maximum amongst the patients classified as the long survivors or low-risk, minimum amongst those classified to be short survivors or high-risk. (B) The top ranked features $(n=47)$ for the high-risk classifier, i.e., the short-survivor patients (left), and the top ranked features $(n=44)$ for the low-risk classifier, i.e., the long-survivor patients (right). 

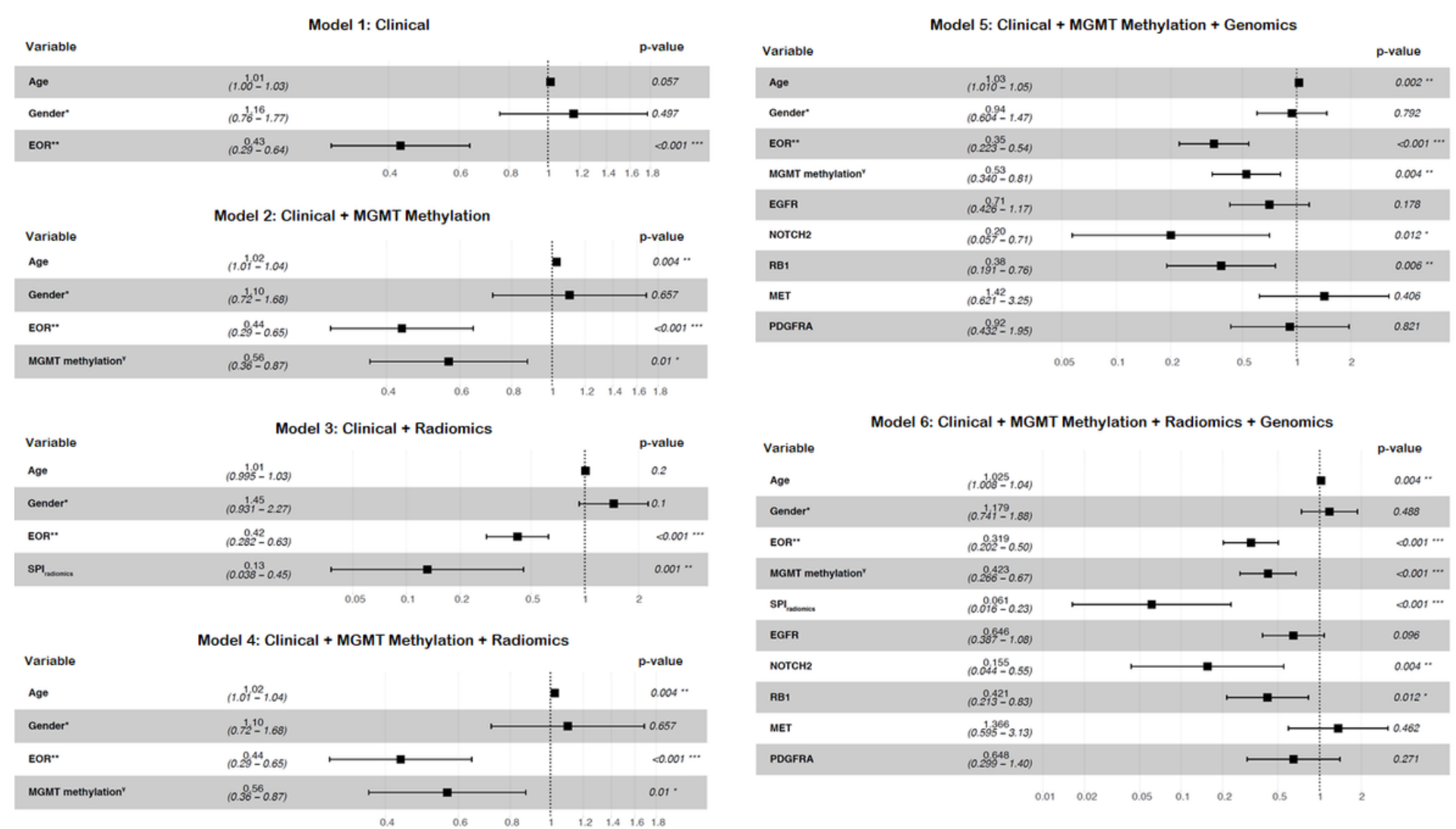

Figure 4

Forest plots for the six presented Cox-PH models, demonstrating the association between each of the covariates and survival. 
Model 1: Clinical

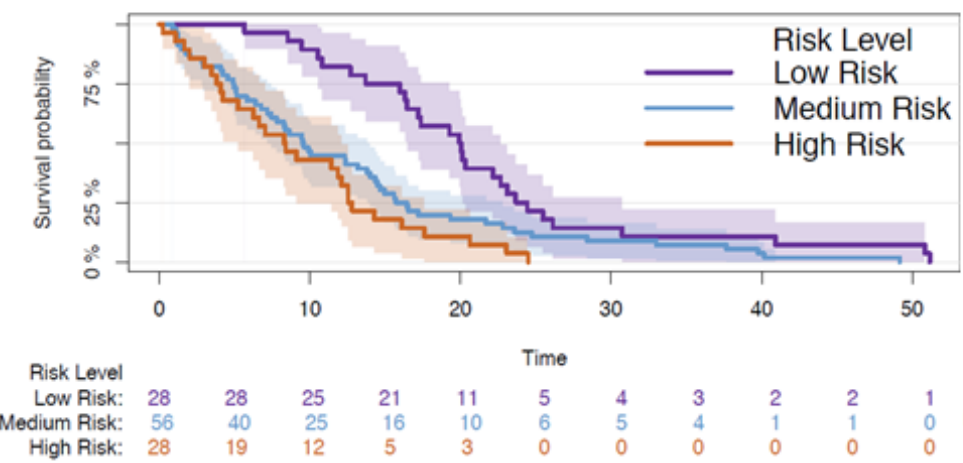

Model 2: Clinical + MGMT

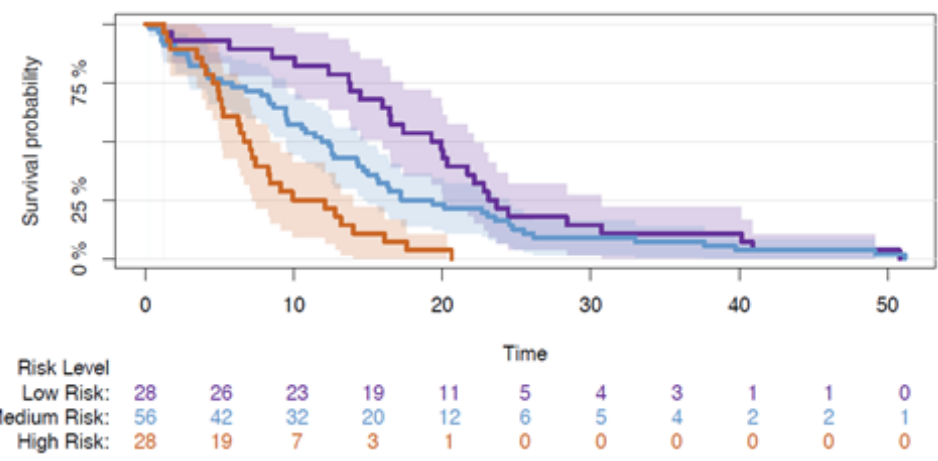

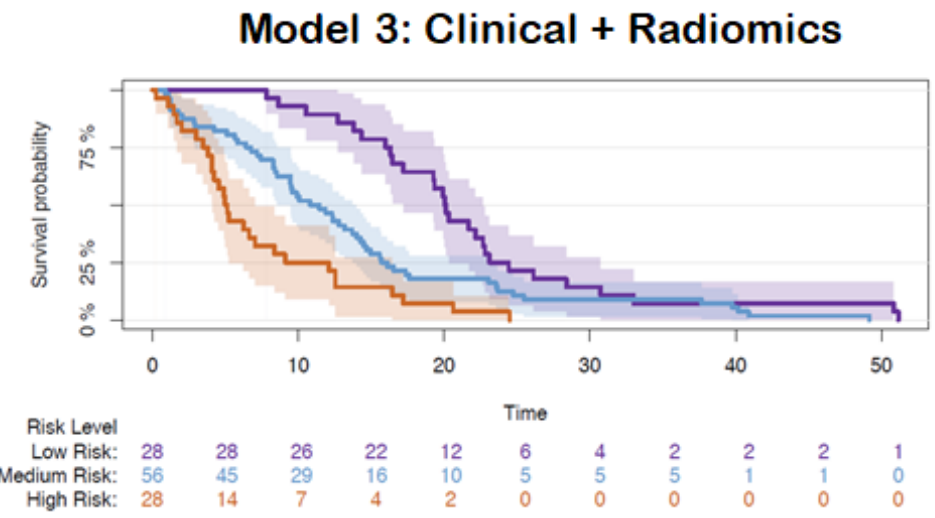

Model 4: Clinical + MGMT + Radiomics

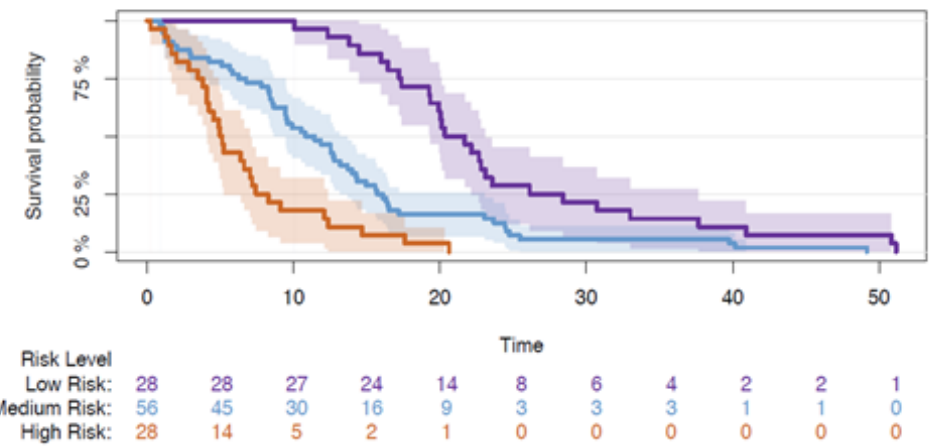

Model 5: Clinical + MGMT + Genomics

Model 6: Clinical + MGMT + Radiomics + Genomics
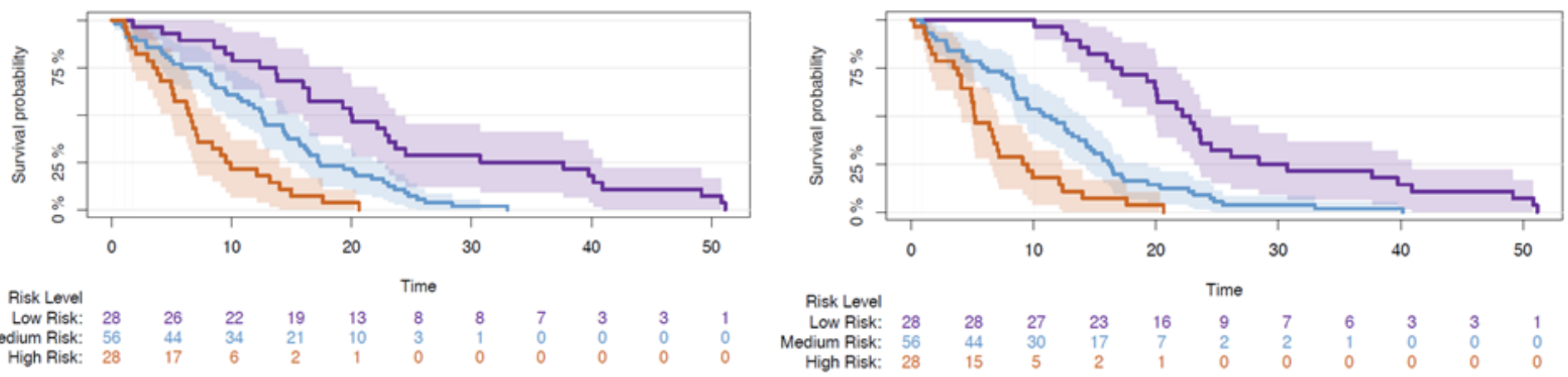

\section{Figure 5}

Risk stratification based on overall survival for the patients in our cohort $(n=112)$ using the six Cox-PH models including different layers of information. For illustration purposes, we have displayed low, medium, and high levels of survival probability. The survival curves are illustrated with their $95 \%$ confidence intervals. 